\title{
Magnetic Response and Negative Refractive Index of metamaterials
}

\author{
Th. Koschny ${ }^{a, b}$, J. Zhou ${ }^{a}$, and C.M. Soukoulis ${ }^{a, b, c}$ \\ ${ }^{a}$ Ames Laboratory \& Department of Physics and Astronomy, \\ Iowa State University, Ames, Iowa 50010, U.S.A. \\ ${ }^{b}$ Institute of Electronic Structure and Laser (IESL) - FORTH, \\ P.O. Box 1527, 71110 Heraklion, Crete, Greece. \\ ${ }^{c}$ Dept. of Materials Science and Technology, University of Crete, Heraklion, Crete, Greece.
}

\begin{abstract}
We study the scaling of negative magnetic response of the SRR from microwave to upper $\mathrm{THz}$ frequencies. We show, that the linear scaling breaks down for SRR sizes below the order of $1 \mu \mathrm{m}$. This breakdown is due to the contribution of the finite electron mass to the inductance of the effective LC oscillator. While at microwave frequencies metals can be treated as near-perfect conductors, close to optical frequencies they rather constitute lossy negative dielectrics. We also study the scaling of the losses in SRR as well as the higher order excitations or plasmon modes and their magnetic response. We discuss the non-resonant diamagnetic response of the SRR and the corresponding corrections to the shape of the frequency dependent effective permeability of the metamaterial. We discuss the connection of recently suggested alternative negative index metamaterial designs in a unified picture.
\end{abstract}

Keywords: SRR, THz, LHM, metamaterial, magnetic response, negative index

\section{INTRODUCTION}

The idea of left-handed (LH) materials, i.e. materials with both negative electrical permittivity $(\epsilon)$ and magnetic permeability $(\mu)$, where the electric field $(E)$, magnetic field $(B)$, and wave vector $(k)$ form a left-handed coordinate system, was developed by Veselago ${ }^{1}$ decades ago. However, it was only recently that such materials were investigated experimentally, ${ }^{2-4}$ despite their rich physics and the large number of associated potential applications, e.g. in the formation of a perfect lens. ${ }^{5}$ The first experimental realization of LH-materials was achieved by separately constructing $\epsilon<0$ and $\mu<0$ components, and then by combining them together forming a left-handed material (LHM). Although it has been well known how to obtain a $\epsilon<0$ material easily (e.g. using wire arrays ${ }^{6}$ ), the realization of $\mu<0$ response (especially at high frequencies) has been a challenge, due to the absence of naturally occurring magnetic materials with negative $\mu$. The possibility of the realization of a $\mu<0$ material was predicted in 1999 by Pendry et al., who suggested a design made of concentric metallic ring with gaps, called split ring resonators (SRRs) which exhibits a $\mu<0$ regime in the vicinity of the magnetic resonance frequency $\omega_{m}$ of the SRR structure. ${ }^{7}$

In the past few years there has been ample proof for the existence of Negative Index Materials (NIMs) in the $\mathrm{GHz}$ frequency range. A lot of groups were able to fabricate ${ }^{2,8-10}$ NIMs with an index of refraction $n=-1$ with losses of less than $1 \mathrm{~dB} / \mathrm{cm}$. Recently different groups observed indirectly ${ }^{11-14}$ negative $\mu$ at the $\mathrm{THz}$ region. In most of the THz experiments ${ }^{11,13,14}$ only one layer of SRRs were fabricated on a substrate and the transmission, $T$, was measured only for propagation perpendicular to the plane of the SRRs, exploiting the coupling of the electric field to the magnetic resonance of the SRR via asymmetry. ${ }^{15}$ This way it is not possible to drive the magnetic permeability negative. Also, no negative $n$ with small imaginary part has been observed yet at the $\mathrm{THz}$ region. ${ }^{16,17}$ One reason is that is very difficult to measure with the existing topology of SRRs and continuous wires both the transmission, $T$, and reflection, $R$, along the direction parallel to the plane of the SRRs. So there is a need for alternative, improved and simplified designs that can be easily fabricated and experimentally

Email address: koschny@ameslab.gov (Th. Koschny). 
characterized. This was recently achieved in the GHz region ${ }^{18,19}$ and in the $\mathrm{THz}$ region ${ }^{16,17}$ by the use of finite length wires and the fish-net topology. Very recent work has moved the negative refractive index at optical wavelengths. ${ }^{20,21}$ Of course, the losses are large in these high frequencies.

In this manuscript, we will present results of the magnetic response of SRRs as the size of SRRs reduce in size. As shown Zhou et. al., ${ }^{22}$ scaling $\left(\omega_{m}\right.$ vs $\left.1 / a\right)$ is obeyed for $a>500 n m$, while for smaller $a$, the magnetic resonance frequency saturates. We have systematically studied the current density as well as the magnetic response of the SRRs. We have also calculated the losses of the SRRs as their size decreases. It is found that losses increase as the size of the SRRs decreases. We have also shown that the effective magnetic permeability $\mu(\omega)$ does not approach 1 as $\omega \rightarrow 0$ and $\omega \rightarrow \infty$. This is due to the diamagnetic response of the SRRs. We present numerical results which support this conclusion. One way to increase the magnetic resonance frequency $\omega_{m}$ is to introduce four-cut SRRs. ${ }^{22}$ We have systematically studied $\omega_{m}$ versus the size of the four-cut SRRs. As in the case of the one-cut SRRs, scaling $\left(\omega_{m} \sim 1 / a\right)$ is obeyed for $a>500 \mathrm{~nm}$ while for $a<500 \mathrm{~nm}$ saturation of $\omega_{m}$ is also obtained. Using the retrieval procedure ${ }^{24,25}$ we have calculated $\mu(\omega)$, both the real and imaginary part of $\mu(\omega)$, for the one-cut and four-cut SRRs. As the size of the SRR ( for both the one-cut and four-cut SRRs) decreases the resonance of $\mu(\omega)$ gets weaker and the imaginary part of $\mu(\omega)$ gets stronger. This also means that the losses become larger as the size of the SRR gets smaller.

\section{SRR MAGNETIC RESPONSE AND SCALING TO THZ FREQUENCIES}

A common constituent to provide magnetic response in metamaterials is the Split-Ring Resonator (SRR). The SRR in its simplest form consists of a highly conductive metallic ring which is broken in one (or several) location(s) by a non-conductive gap of air or other dielectric materials. If this ring is placed in a temporally varying magnetic field an electric circular current is induced in the metallic ring which in turn leads to charge accumulating across the gaps. The electric field which builds due to the charge at the gap counteracts the circular current leading to oscillations of the current in the SRR ring in which energy is converted back and forth between the electric field energy stored (predominantly) in the vicinity of the gaps and magnetic field energy concentrated in the region enclosed by the ring. The SRR is thus a resonator which couples to a perpendicular magnetic field and can be characterized by the effective capacitance of the gap(s) and effective inductance of the loop define by the ring. It can be understood in terms of a resonant LC circuit with a resonance frequency $\omega_{m}^{2}=1 / L C$, where $L$ is the inductance and $C$ is the the capacitance of the SRR. The resonant response of the circular current in the SRR to an external magnetic field leads to a resonant magnetic moment which may reach large negative values for a relatively small frequency region just above the resonance frequency. A metamaterial made up of a periodic array of SRRs such that the size of the SRR is much smaller than the wavelength of an incident electromagnetic wave around the resonance frequency behaves as a homogeneous effective medium with a negative (resonant) permittivity $\mu_{\mathrm{eff}}(\omega)$.

\subsection{Scaling of the resonance frequency}

SRRs were first employed at microwave frequencies, i.e. at some tens of GHz. In this region the metals which make up the SRR ring have a very high conductivity, hence a large essentially imaginary permittivity, and can be considered near-perfect conductors which exclude all electric field from the volume of the metallic ring. As a consequence, the resonance of the SRR is entirely determined by the geometry of the SRR and the (essentially frequency independent) dielectric properties of the surrounding materials like the substrate that carries the SRR rings. Therefore, for a given SRR size, $a$, the resonance frequency, $\omega_{m}$, scales as $1 / a$, keeping the vacuum speed of light invariant. This conveniently allows to shift the negative permittivity band of the SRR metamaterial just shrinking the structural size of the SRR throughout the microwave region. As shown by Zhou et al. ${ }^{22,23}$ this linear scaling does not extend into the upper $\mathrm{THz}$ and optical region. Instead, $\omega_{m}$ saturates if the structural size is decreased to the order of some $500 \mathrm{~nm}$. The saturation of $\omega_{m}$ is due to the contribution of the kinetic energy of the moving electrons in the SRR ring to the energy stored in the SRR resonance which, at high frequencies, becomes comparable to the energy stored in the magnetic field. This kinetic energy, $E_{k}=\left(n_{e} V\right) m_{e} v_{e}^{2} / 2=L_{e} I^{2} / 2$ (here, $n_{e}$ is the electron density, $V$ the volume carrying the current, $I$, and $m_{e}$ the electron mass and $v_{e}$ the electron drift velocity, i.e. proportional to the current) can be identified with an additional inductance $L_{e}$ and adds to the magnetic field energy, $E_{m}=L_{m} I^{2} / 2$, given by the geometric inductance $L_{m}$ which dominates the 

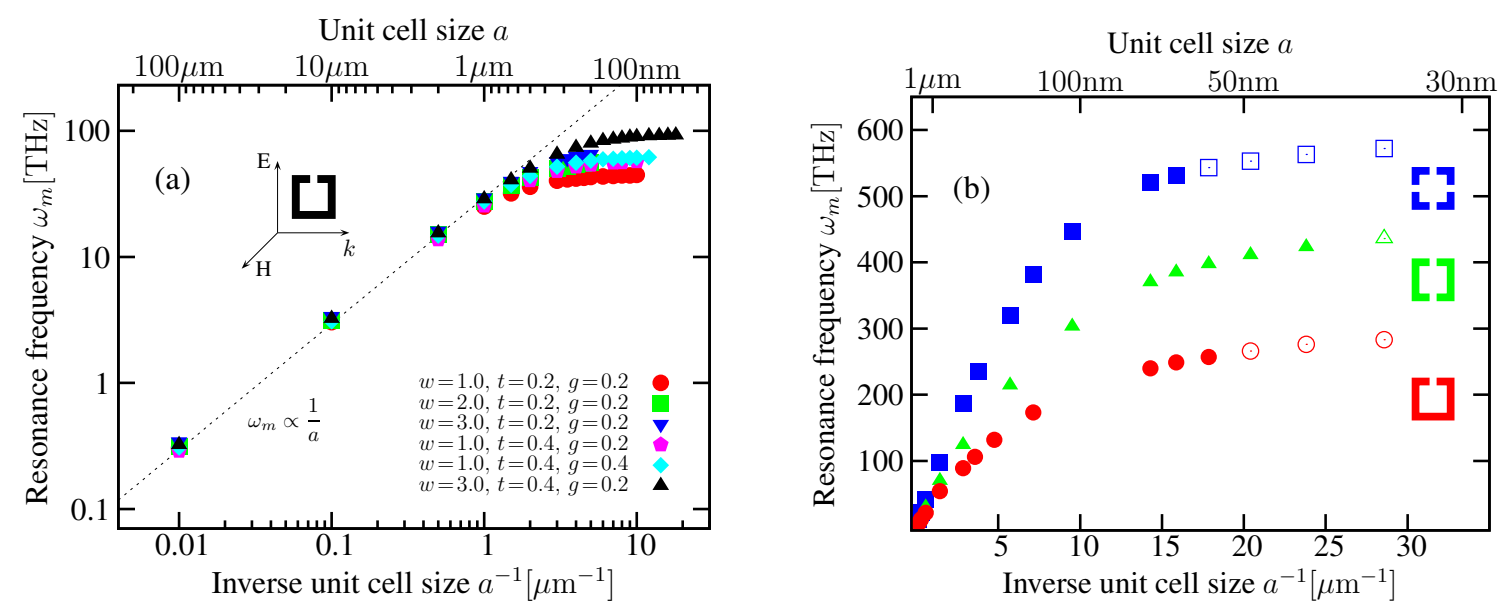

Figure 1. Scaling of the magnetic resonance frequency $\omega_{m}$ with the linear size of the SRR. (a) Scaling for single-gap SRR for different ring widths $w$, thicknesses $t$, gap width $g$, and fixed side length $l=0.8$ in units of the unit cell size $a$; (b) Scaling for one-, two-, and four-gap SRR of equal size in comparison. The SRR metal is gold, described by a Drude model dielectric function $\left(\omega_{p}=2175 \mathrm{THz}, \omega_{\tau}=6.5 \mathrm{THz}\right)$.

scene at low frequencies. Careful analysis shows that $L_{e}$ scales like $\sim 1 / a$ with the linear structural size of the SRR while the geometric inductance, $L_{m}$, and the geometric capacitance, $C$, contributed by the gap(s) in the SRR ring scale $\sim a$. Thus the magnetic resonance frequency, $\omega_{m}=\left[\left(L_{e}+L_{m}\right) C\right]^{-1 / 2} \sim\left[1+\text { const } a^{2}\right]^{-1 / 2}$, has to saturate to a constant value for small enough structure size $a \rightarrow 0$. To a smaller extend, also the capacitance has to be corrected to account for the polarization of the SRR ring. ${ }^{27}$

The saturation of $\omega_{m}$ sets limits to the geometric scaling of the magnetic resonance to higher frequencies towards the optical region. However, the actual limiting frequency strongly depends on the actual parameters of the SRR. In Fig 1, we present simulation results obtained for single-ring SRRs similar to the designs previously used in experiments in the $100 \mathrm{THz}$ range. ${ }^{13}$ The propagation direction of the incident EM wave is in the SRR plane with the magnetic field perpendicular to the SRR ring, driving the magnetic resonance. The electric field is chosen in the symmetry direction of the SRR to avoid electric coupling to the magnetic resonance. ${ }^{15}$ In Fig. 1a, the scaling of single-gap SRRs with different values for ring width, metal thickness and gap width are shown. The linear scaling up to lower THz frequencies and SRR dimensions of just below the order of $1 \mu \mathrm{m}$ is clearly visible. For smaller SRR sizes the resonance frequency starts to saturate, reaching its limiting value for SRR sizes of about $50 \ldots 100 \mathrm{~nm}$. The actual value of the limiting frequency, however, shows some significant variability, reaching (for the chosen examples) from 30 to 100THz. Higher saturation values for $\omega_{m}$ at a given structural size of the SRR can be achieved increasing the gap size, the ring width, or the thickness of the metal trace; i.e. just parameters which increased the resonance frequency of the SRR in the microwave regime. ${ }^{28}$ An alternative possibility to increase the limit of $\omega_{m}$ is to increase the number of gaps in the SRR ring as shown in Fig. 1b. For the two- and four-gap SRR our simulations show negative magnetic response up into the optical region $(500 \mathrm{THz}$ for 4-gap), although the minimum of the effective permeability is very close to zero ( $\operatorname{Re} \mu \approx-0.1$ ) in this case and the losses substantial $(\operatorname{Im} \mu \approx 0.5)$.

In Fig. 2, we present numerical data for the real and the imaginary part of the effective magnetic permeability $\mu(\omega)$ for the scaling of a single-gap SRR from a (linear) unit cell size of $100 \mu \mathrm{m}$ down to $50 \mathrm{~nm}$. Notice that the resonant response of $\mu(\omega)$ does not change much for sizes $>1 \mu \mathrm{m}$, i.e. while the linear scaling of the resonance frequency $\omega_{m}$ holds, but gets rapidly weaker as the size of the system decreases in the nanometer region. Notice also that the effective permeability of the single-cut SRR approaches unity for high frequencies where the resonant response dies out. For the lower frequency cases we observe that the $\mu(\omega)$ curves start for frequencies smaller the corresponding $\omega_{m}$ slightly below unity and do not symmetrically return to unity above $\omega_{m}$. This is the diamagnetic response of the SRR and will be discussed below. Also the peak in the imaginary part of $\mu(\omega)$ gets smaller, and its width increases as the size of the SRR gets smaller. 

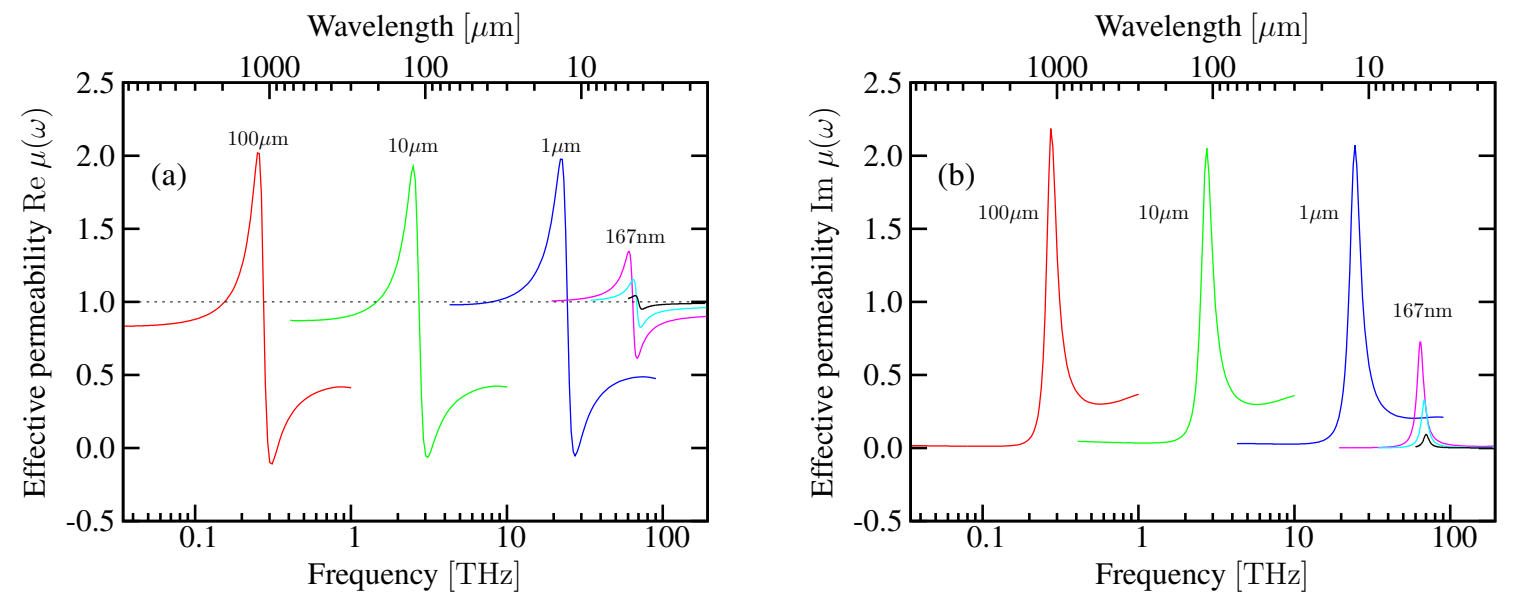

Figure 2. Scaling of the real (a) and imaginary (b) part of the retrieved effective permeability for different sizes $a$ of the SRR unit cell: $a=100 \mu \mathrm{m}$ (red), $10 \mu \mathrm{m}$ (green), $1 \mu \mathrm{m}$ (blue), $167 \mathrm{~nm}$ (pink), 100nm (turquoise), and 50nm (black). The SRR metal is given by a Drude model for gold.
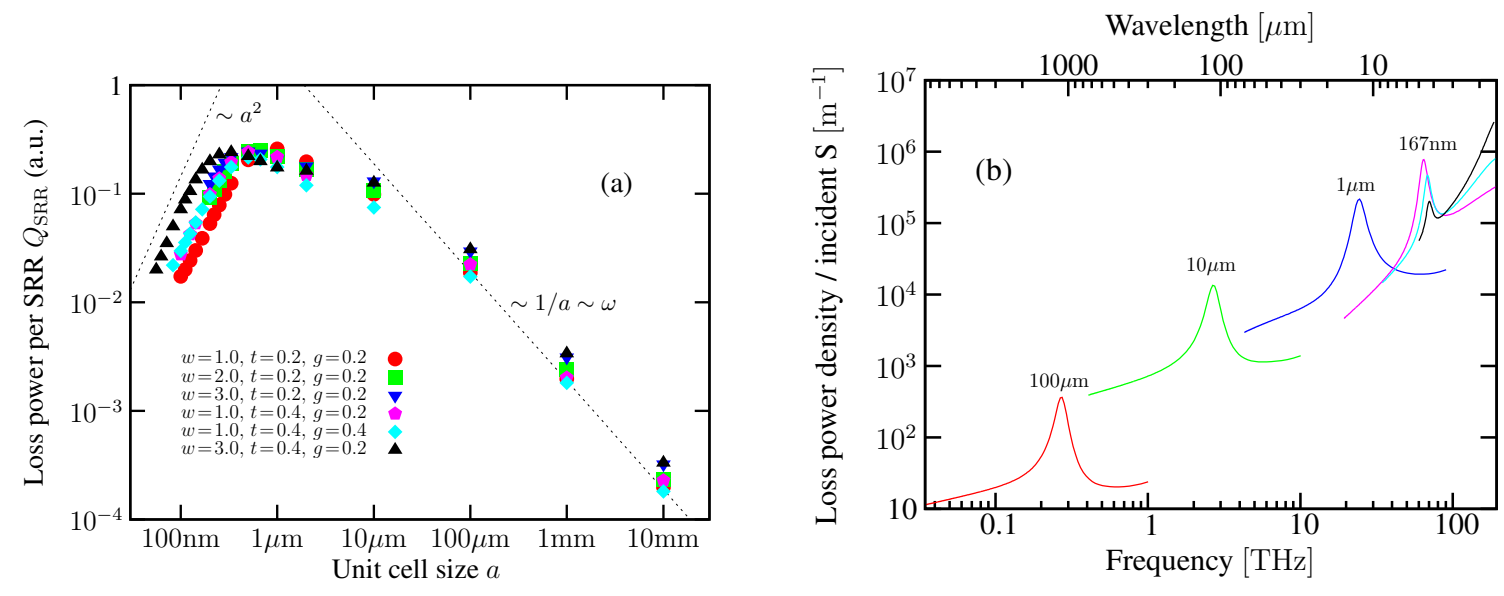

Figure 3. Scaling of losses: (a) Scaling of absorption in single unit cell with unit cell size (for unit energy influx to the unit cell) for the same SRR geometries as shown in Fig. 1a; (b) Loss power density per incident Poynting vector $S$ as function of frequency for the same SRR unit cell sizes as shown in Fig. 2. 

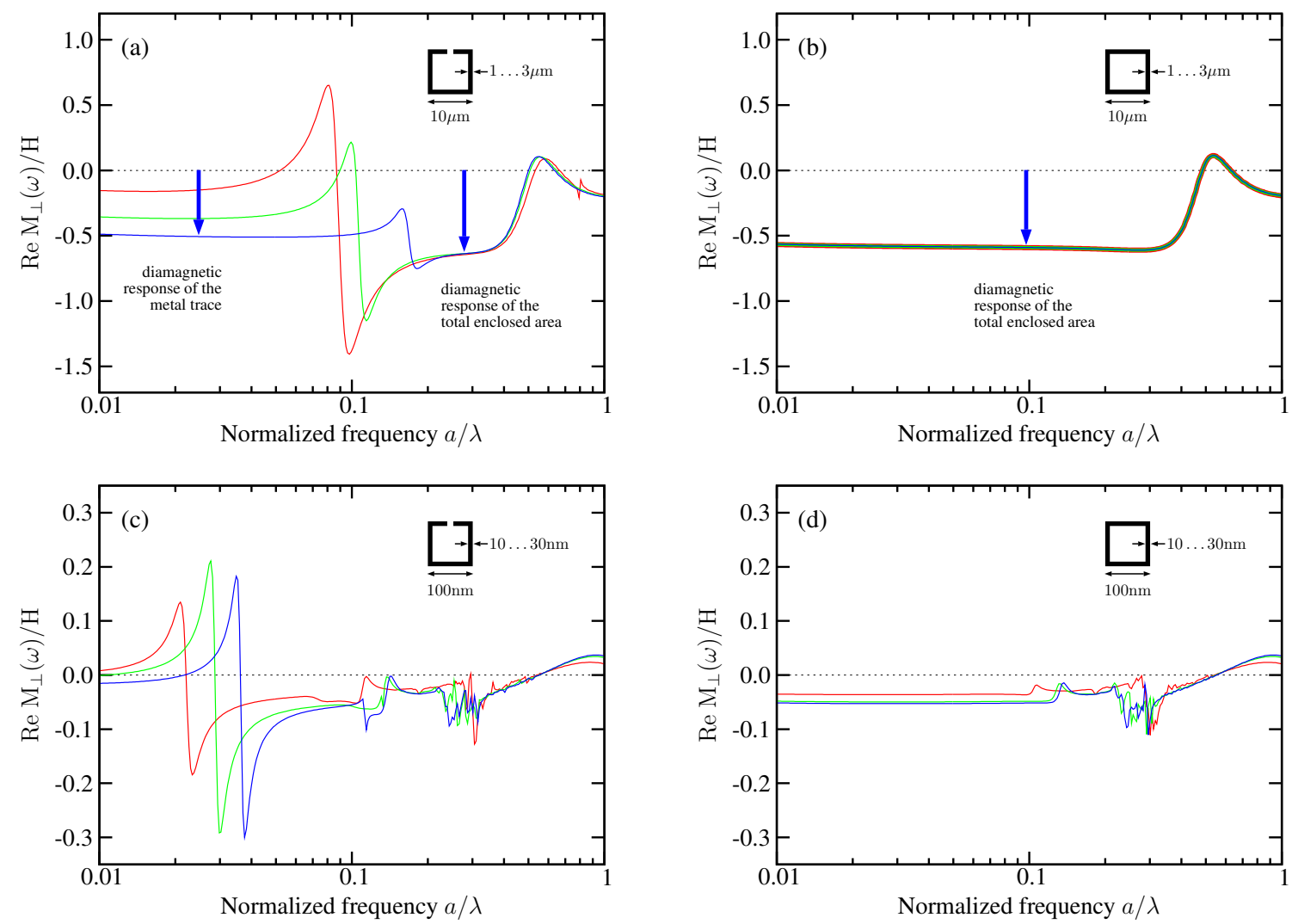

Figure 4. Magnetic moment as a function of frequency for open and closed SRR at $10 \mu \mathrm{m}$ and $100 \mathrm{~nm}$ unit cell size. Dependence on the SRR ring width: $w=1 \mu \mathrm{m}$ (red), $2 \mu \mathrm{m}$ (green), $3 \mu \mathrm{m}$ (blue) for the $10 \mu \mathrm{m}$ unit cell size open (a) and closed (b) SRR; $w=10 \mathrm{~nm}$ (red), 20nm (green), 30nm (blue) for the 100nm unit cell size open (c) and closed (d) SRR.

\subsection{Scaling of losses}

Another important parameter is the loss in the SRR and its scaling by decreasing the SRR size. The loss is related to the loss peak of the imaginary part of the effective magnetic response $\mu(\omega)$. In our simulation we have the advantage to have access to the local EM fields and can therefor calculate the loss power given by the ohmic loss directly as $P=\int \mathbf{j} \cdot \mathbf{E} d V$, where the current density $\mathbf{j}$ is given by $\sigma \mathbf{E}+\partial \mathbf{D} / \partial t$ and the material properties $\varepsilon_{\text {metal }}(\omega)$ of the SRR ring. In Fig. 3a, we show the scaling of the total (peak) loss power per SRR unit cell with the size of the SRR from microwave to upper $\mathrm{THz}$ frequencies for some of the the single-gap SRR designs already employed in Fig. 1a. It is clearly visible that for low frequencies the loss power per SRR increases inverse proportional to the SRR size, i.e. the loss per SRR increases linearly with the resonance frequency $\omega_{m}$. In the region where $\omega_{m}$ saturates the loss power per SRR actually starts to decrease again. However, this is only due to the vanishing resonances strength of the magnetic resonance as can be seen from Fig. 2a and is not helpful in achieving lower loss metamaterials. Fig. 3b shows the behavior of the normalized loss power density per incident Poynting vector (energy current density) for the single-gap SRR cases shown in Fig. 2. Also here the linear increase of the loss with frequency for larger SRR sizes is evident while in the saturation region the resonance dies out, effectively reducing the absorption. Note that not only the loss peak but also the non-resonant loss around $\omega_{m}$ increases at least linearly with omega and starts to dominate the total loss when the resonance dies out and the associated absorption peak becomes small.

\subsection{Resonant magnetic moment and diamagnetic response}

The magnetic response of the SRR metamaterial is usually characterized by its effective permeability $\mu(\omega)$. This can be obtained via a retrieval procedure ${ }^{25}$ form the plane wave scattering amplitudes of a finite slab of 

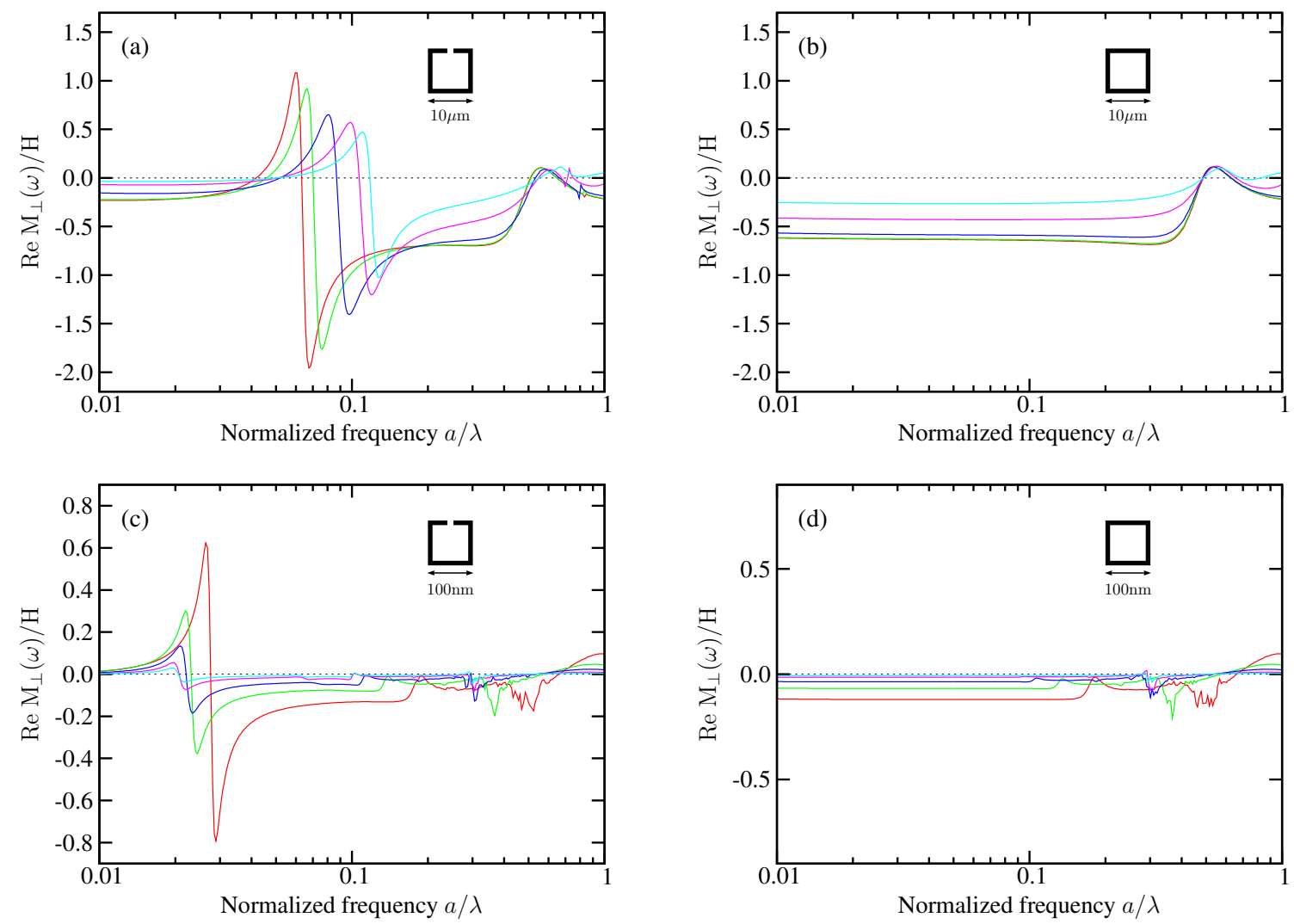

Figure 5. Magnetic moment for open and closed SRR at $10 \mu \mathrm{m}$ and $100 \mathrm{~nm}$ unit cell size $a$. Dependence on ring separation $d$ perpendicular to SRR plane in units of the unit cell size: $d=1 / 20$ (red), 1/10 (green), 1/5 (blue), 1/2 (pink), and 1 (turquoise) for open $(\mathrm{a}, \mathrm{c})$ and closed $(\mathrm{b}, \mathrm{d}) \mathrm{SRR}$.

metamaterial. In simulations where we have access to the local fields we can alternatively calculate the magnetic moment density directly as (1/2) $\int \mathbf{r} \times \mathbf{j} d V$ with the current density $\mathbf{j}$ given by $\sigma \mathbf{E}+\partial \mathbf{D} / \partial t$. In Fig. 4 , we present the calculated magnetic moment for SRRs and closed SRRs of different ring widths at a structural size of $10 \mu \mathrm{m}$ (corresponding to a resonance frequency of $\omega_{m} \approx 3 \mathrm{THz}$ which is still in the linear scaling regime) and of $100 \mathrm{~nm}$ (corresponding to $\omega_{m} \approx 70 \mathrm{THz}$ and deep within the saturation regime for this geometry). For the SRRs we observe the expected resonant shape of the real part (and imaginary part, not shown) of the magnetic moment corresponding to the resonant behavior observed in $\mu(\omega)$. If the gaps in the SRR are closed, the magnetic resonance disappears as expected from the LC oscillator model. However in contrast to the simple resonance model where the magnetic moment should approach zero below and above the resonance frequency, we observe a saturation of $M$ at a substantial negative value below and above the resonance. The shift is larger for $\omega>\omega_{m}$ than for $\omega<\omega_{m}$, but also occurs for the closed SRR. It can be explained by the diamagnetic response of the SRR.

For microwave frequencies the metal of the SRR ring is a near perfect conductor. The SRR responds to the external magnetic field with a circular current around the ring polarizing the gap. This is the LC resonator. The current can flow only in a thin layer near the surface extending up to the order of the skin depth $\delta_{s}(\omega)$ into the metal. At low frequency (good conductor) the skin depth is dominated by the imaginary part of the metal's dielectric functions and is given by $\delta_{s}(\omega) \approx\left(\sigma \mu_{o} \omega\right)^{-1 / 2}$, where $\sigma$ is the DC conductivity of the metal. Because of the scaling $\delta_{s} \sim \omega_{m}^{-1 / 2} \sim a^{1 / 2}$ near the resonance the ratio of this surface layer to the width of the SRR ring (width $\sim a$ ) becomes $\sim a^{-1 / 2}$ and is small in the microwave region (typically $1 / 10$ th of the ring width). This allows for an additional current in response to the external magnetic field which flows around the edges of the 
SRR metallic ring (outer and inner circumference and along the faces of the gap) in the plane perpendicular to the external magnetic field, expelling the latter from the area of the metal of the ring. This is the diamagnetic response of the metallic ring. The associated negative magnetic moment leads to the shift observed for $\omega<\omega_{m}$. While for frequencies below the resonance the impedance of the gap is large, it becomes small for frequencies well above the resonance. This allows for the diamagnetic current to flow now at the outer circumference of the ring and "across" the gap, leading to exclusion of the magnetic field from the entire enclosed area of the SRR and hence a stronger negative magnetic moment and diamagnetic response. This second type of diamagnetic response is also expected from the closed metallic ring and exactly what we see for the closed SRR's magnetic moment in Figs. 4 and 5. The diamagnetic response due to the metallic area increases with increasing ring width as shown in Fig. 4a. In contrast the diamagnetic response of the entire enclosed area for $\omega>\omega_{m}$ in Fig. 4a and all $\omega$ in Fig. $4 \mathrm{~b}$ is essentially independent on the ring width. Note that the structures above the magnetic resonance are due to higher (electric and magnetic) resonances of the SRR and due to periodicity band structure effects when the wave length becomes comparable to the SRR unit cell size and will not be discussed here.

For higher THz frequencies the metals of the SRR ring can not be considered near perfect conductors anymore. The dielectric function of the metal starts to be dominated by its real part. Consequently the previously used approximation of the skin depth breaks down and has to be replaced by $\delta_{s}=(c / \omega) \operatorname{Im}\left[\varepsilon_{\text {metal }}(\omega)\right]^{-1 / 2}$ which saturates to a value of the order of $10 \mathrm{~nm}$ at optical frequencies. That means, scaling the dimension of the SRR down beyond the order of $1 \mu \mathrm{m}$ the skin depth increases relatively to the width of the SRR such that the first kind diamagnetic currents (which were flowing anti-parallel on the inner and outer edge of the ring) get suppressed. Hence we still see much less diamagnetism below $\omega_{m}$ in Fig. 4c, and the negative magnetic moment is stronger for a wider ring. Above the resonance and for the closed ring in Fig. 4d we see essentially the same behavior as explained above. Only the higher loss leads to a weaker diamagnetic response. Note that due to the saturation of $\omega_{m}$ the resonance moves to smaller $a / \lambda$.

In Fig. 5 we present the dependence of the magnetic moment of the SRR on the density of SRRs in the direction perpendicular to the SRR plane. Stacking the SRRs closer basically has two effects: $(i)$ the magnetic moment per volume increases leading to a larger amplitude of the magnetic resonance and stronger diamagnetism, and $(i i)$ the resonance frequency $\omega_{m}$ reduces because of the enhancement of the loop inductance of the SRR ring due to the mutual inductance of neighboring rings in stacking direction (Fig. 5a). As one would expect, for a strong magnetic response of the SRR metamaterial a dense packing of the SRRs is desirable. In Fig. 5b, we observer the strange behavior that increasing the perpendicular separation of the ring the resonance frequency $\omega_{m}$ does slightly decrease as opposed to the expected increase which is seen for larger structures (Fig. 5a). This may be explained by noticing that all cases in Fig. $5 \mathrm{~b}$ are deeply in the saturation regime for $\omega_{m}$ if now the geometric inductance is enhanced by moving the rings closer, the saturation $\left(L_{e} \gg L_{m}\right)$ is a little bit "postponed" and $\omega_{m}$ moves slightly higher, i.e. closer to the extrapolated linear scaling curve $\omega_{m} \sim 1 / a$.

\subsection{Higher order modes}

For microwave frequencies the LC resonator circuit picture is a good approximation of the SRR. The main reason for this is that at such low frequencies we can neglect the (real part of the) polarization of the metal due to the huge conductivity and assume that charges accumulated in the very small area of the faces of the gap(s) and the current is spatially constant along the ring. At higher $\mathrm{THz}$ frequencies those assumptions are no longer valid. Here the current has to be treated as a charge density wave along the SRR and shows plasmonic resonances. As a very crude picture we could imagine the resonances of the SRR as charge density waves on a rod of a length equal to the arc length of the SRR ring. This rod supports plasmonic modes (plasmonic means in this situation that the inductance is coming from the electron mass and the capacitance from the external electric field over the surface of the rod) which occur at discrete frequencies whenever we have current nodes at the ends of the rod. This picture is of limited value: $(i)$ the EM response of the modes, especially the classification of electric vs. magnetic response, depends on the geometry and is entirely different for a U shaped SRR than for a straight rod although the qualitative current distribution over the arc length is equivalent; $(i i)$ the plasmonic dispersion depends on the geometric inductance, i.e. the energy stored in the external field outside the metal, which is much larger for the SRR than for the rod and spatially non-uniform along the SRR ring; (iii) the modes of coupling are different. Nevertheless, considering the current distribution is an essential tool for understanding the resonant 

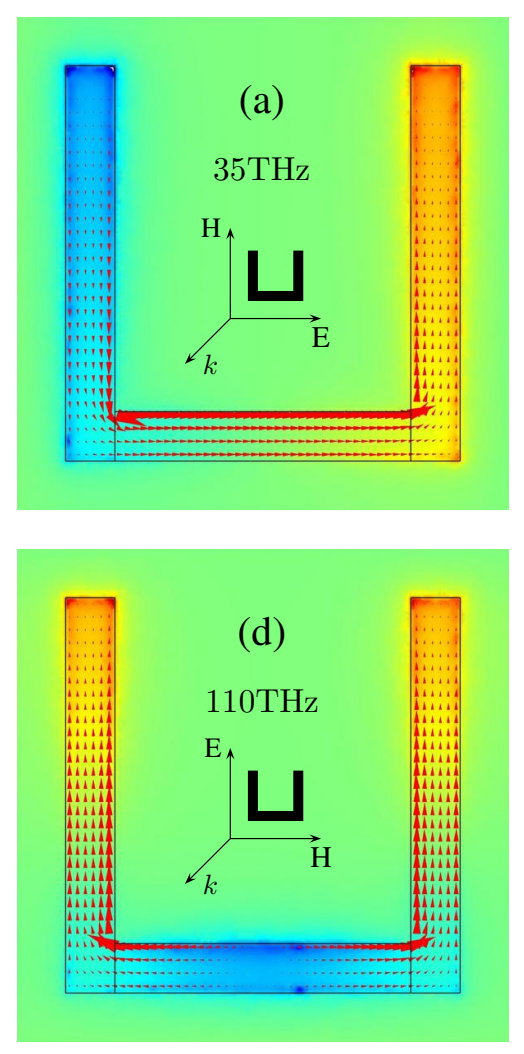
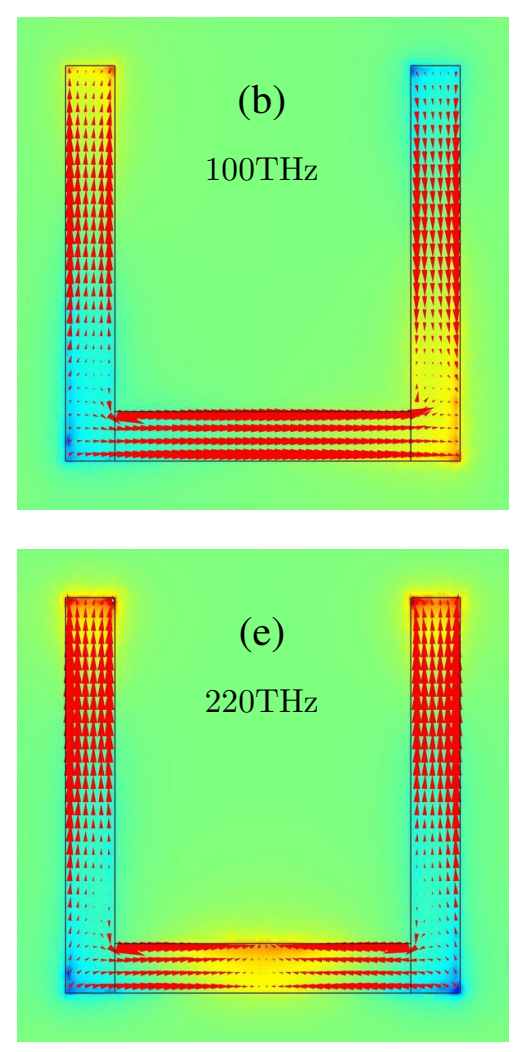
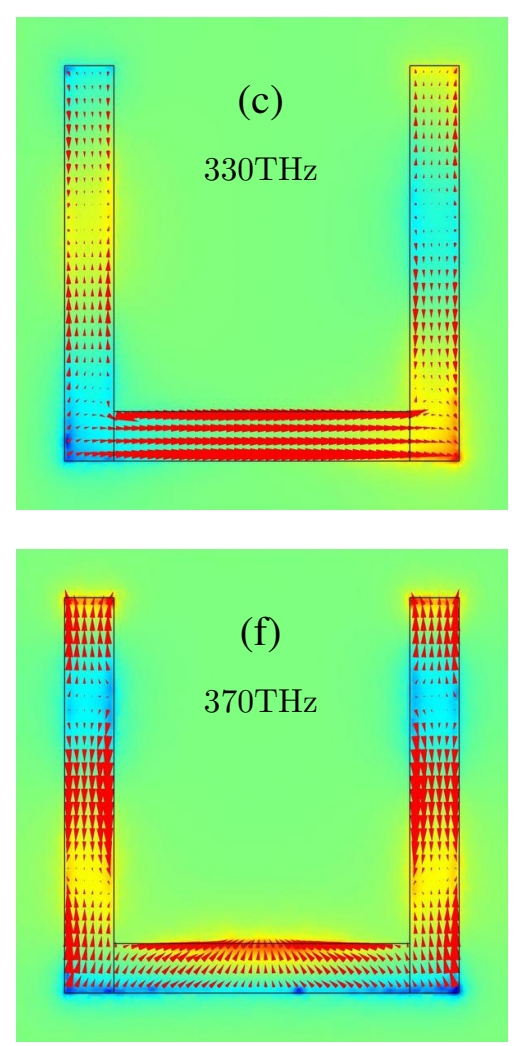

Figure 6. Distribution of the perpendicular component of the surface electric field (color scale; red positive, blue negative) and the bulk current density (arrows) for the lowest few resonant modes of the SRR. The SRR metal is made of a Drude model for gold $\left(\omega_{p}=2175 \mathrm{THz}, \omega_{\tau}=6.5 \mathrm{THz}\right)$, the geometry parameters are: $a_{x}=a_{y}=1 \mu \mathrm{m}, a_{z}=200 \mathrm{~nm}$ (unit cell size), $l_{x}=l_{y}=800 \mathrm{~nm}$ (arm length), $w=100 \mathrm{~nm}, t=50 \mathrm{~nm}$ (ring width and thickness, respectively). The current distributions are shown temporally $\pi / 2$ phase shifted against the charge distribution.

modes of a SRR at higher $\mathrm{THz}$ frequencies. In the microwave region only the remnants of the lowest order mode survive.

Fig. 6 shows the distribution of the current and charge density for the lowest three resonant modes of a $U$ shaped SRR of $800 \mathrm{~nm}$ side length. The current is obtained directly from the simulation, the charge density relates to the perpendicular electric field at the surface of the metal. All fields are time harmonic; the current distributions are shown temporally $\pi / 2$ phase shifted against the charge distribution. The first three panels $(a, b, c)$ show the lowest three resonances for normal incidence to the SRR with the electric field breaking the symmetry of the SRR and thus coupling the the "magnetic" resonance. ${ }^{15}$ The distribution is qualitatively equivalent to the pure magnetic coupling (i.e. propagation in the SRR plane with perpendicular magnetic field), which case is however hard to realize experimentally at such high THz frequencies. All three modes have nonzero magnetic moment coming from all three "arms" of the SRR. The number of current nodes (where charge accumulates) increases with the resonance frequency from two (fundamental mode, corresponding to LC circuit), one on either side of the "gap", to four and six, which have additional nodes inside the continuous metal. The electric excitation of these modes occurs via the polarization of the bottom arm of the SRR by the incident EM wave. For a straight rod these SRR modes would correspond to the $\lambda / 2,3 \lambda / 2$, and $5 \lambda / 2$ mode; for the rod there is however no magnetic moment associated with them. Note that these modes also expose an electric dipole moment; therefore the SRR has a combined magnetic and electric response in this configuration. For the other polarization, normal incidence to the SRR with the electric field along the symmetry axis of the SRR, shown in panels $(\mathrm{d}, \mathrm{e}, \mathrm{f})$ we see the analog plasmonic modes with three, five and seven current nodes; all of which reflect 

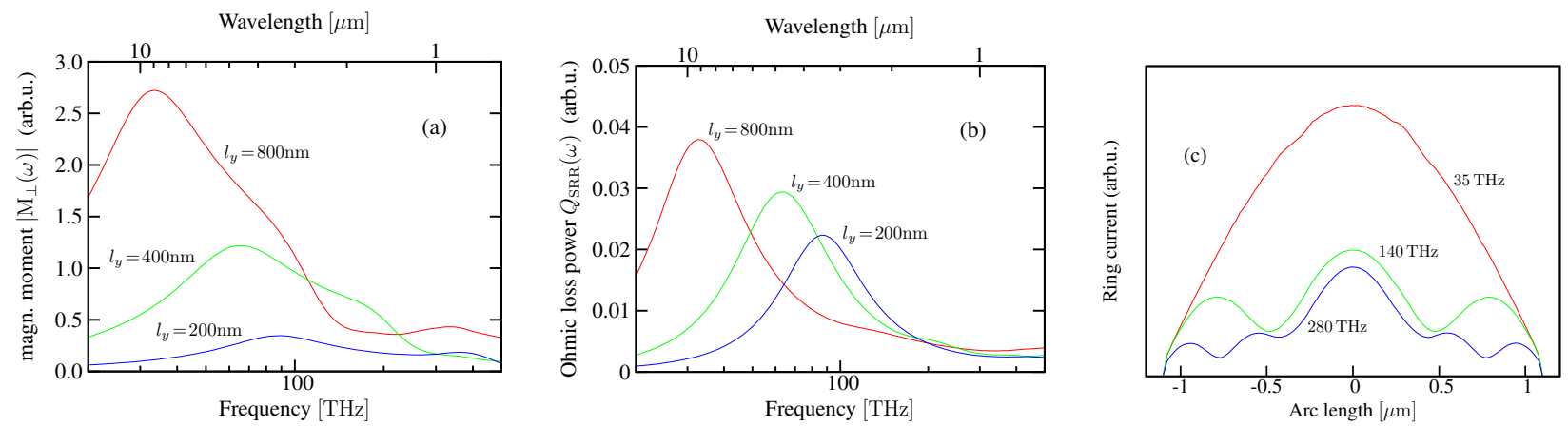

Figure 7. Magnetic moment (a) and Ohmic loss (b) as function of frequency for normal incidence to the SRR plane. The electric field direction is parallel to the cut side, such that it can excite the magnetic resonance. The current distribution (c) along the metal ring of the SRR as function of the arc length. The SRR geometry is the same as in Fig. 7a, except that the arm length $l_{y}$ varies as indicated in panels $(\mathrm{a}, \mathrm{b})$.

the mirror symmetry of the SRR and can thus have no magnetic moment. For the rod, they would correspond to the even, $\lambda, 2 \lambda$, and $3 \lambda$ modes (which cannot be exited for the rod because of their vanishing dipole moment). In the case of the SRR they do possess electric dipole moment in the E-direction, i.e. along the two parallel arms of the SRR, and represent a purely electric response of the SRR.

In Fig. 7, we show the magnetic moment (a) and the loss power (b) for the magnetic resonant modes of the SRR as a function of frequency for three different U shaped SRRs with different lengths of the two parallel arms. It is clearly seen that the lowest order magnetic resonance provides the strongest magnetic response; but also the higher modes have non-zero magnetic moment. As one expects, reducing the length of the parallel arms reduces the magnetic moment. It also shifts the resonances to higher frequencies. This is a combined effect of shortened arc length and reduced geometric loop inductance. In the limit of only the bottom arm left (i.e. a straight rod) the magnetic response would vanish. Note, that the loss decreases much slower with decreasing arm length than the magnetic moment, reconfirming the importance of loop area for strong magnetic response which was well known form the microwave designs.

Fig. 7c shows the distribution of the total current (current density $\mathbf{j}$ integrated over the cross-section of the SRR ring) over the arc length around the SRR ring for the three resonant modes in Fig. $6(\mathrm{a}, \mathrm{b}, \mathrm{c})$. We clearly see the different number of current nodes, which do not reach zero (for the higher modes) because of the superposition with the non-resonant response of the lower order modes (which are fairly broad due to the high losses). Also note the non uniform spacing of the nodes for the higher orders which is different from the straight rod and due to the curvature of the SRR and the coupling to the bottom arm only.

\section{ALTERNATIVE STRUCTURES}

Although the SRR is well suited as a local magnetic resonator in metamaterials and can be scaled, although with limitations, up to near-optical frequencies it has the disadvantage that by itself it does not provide a negative refractive index. To obtain a negative effective refractive index $n_{\text {eff }}(\omega)$ we need, in some finite frequency interval, simultaneously negative (and essentially real) values of the effective permeability $\mu(\omega)$ and permittivity $\varepsilon(\omega)$; Hence a simultaneous negative magnetic and electric response is needed. In the microwave region, a negative permittivity is usually achieved by combining the SRRs with an array of thin continuous wires which provide a plasmonic $\varepsilon(\omega)$ with effective plasma frequencies in the microwave region. This approach is not well suited for $\mathrm{THz}$ frequencies because of dimensional problems and the absence of good conductors. Also the SRR itself has been shown to expose an electric resonance (analog to that of a short piece of straight wire) that can provide a resonant negative effective $\varepsilon(\omega)$, similarly to the resonant $\mu(\omega)$ of the magnetic resonance. This electric resonant response of the SRR is much stronger than the magnetic response; however, due to the principal geometry of the SRR it (almost) always occurs well above the magnetic resonance frequency $\omega_{m}$ such that there is virtually no chance to make the negative $\mu(\omega)$ and $\varepsilon(\omega)$ regions overlap. (because the $\varepsilon(\omega)$ resonance is much stronger and 

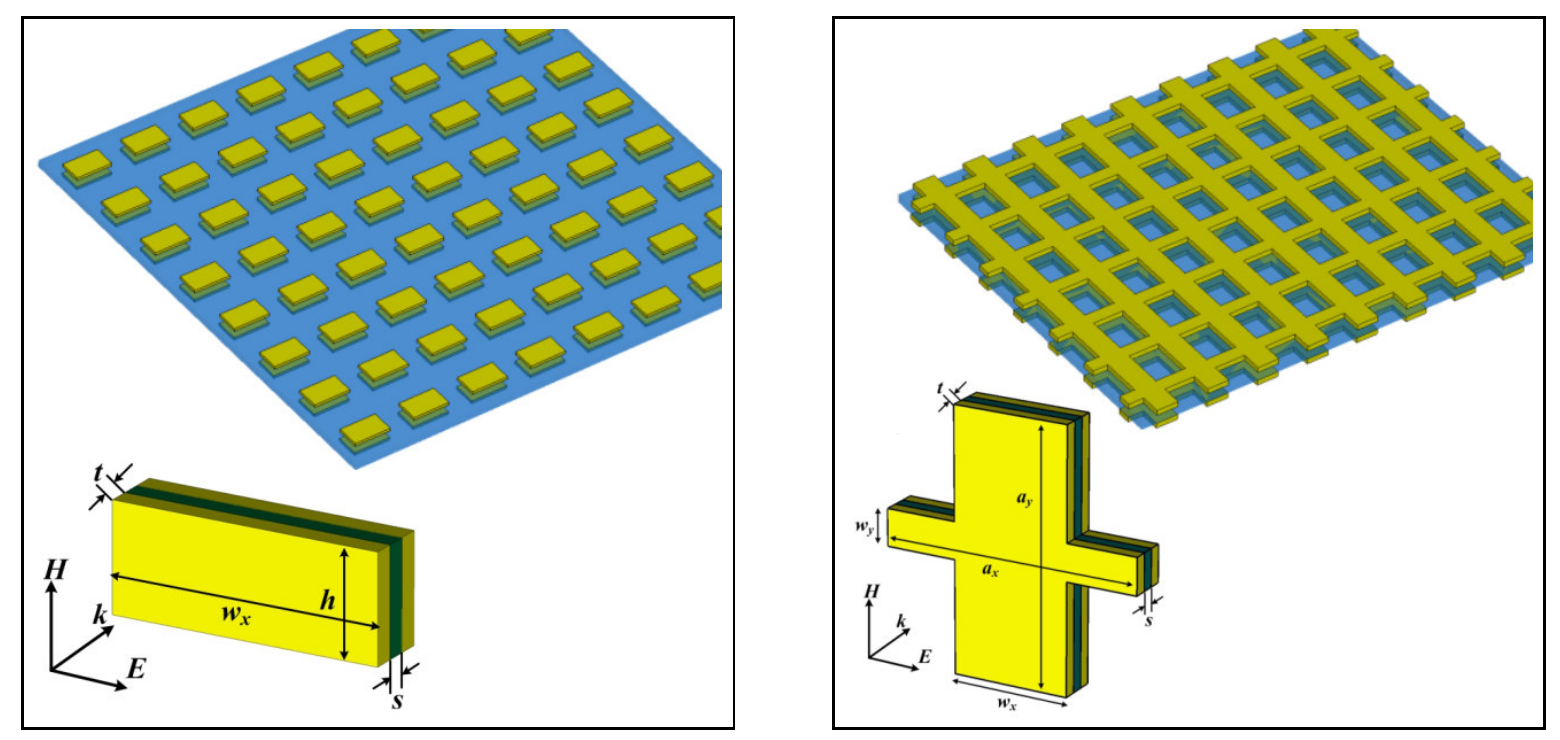

Figure 8. Geometries for short-wire pair arrays and the fishnet structure. Both consist of a patterned metallic double layer (yellow, usually $\mathrm{Au}$ ) separated by a thin dielectric layer (blue).

the $\varepsilon<0$ region therefore wider, we would have to move the $\mu(\omega)$ resonance into the negative permittivity region of the electric resonance.) Alternatively, a pair of parallel short wires can be uses as a magnetic resonator. ${ }^{30}$ This geometry can be viewed as an extremal case of a two-gap SRR ring where the two continuous arms become long but finite while the gap-bearing arms become short and the gaps wide. This limiting case of the two-gap SRR in principle can show both magnetic (resonant circular "ring" current) and electric (resonant linear current parallel in both continuous arms) resonances; because of the greatly reduced loop inductance, here $\omega_{m}$ increases and can move very close to the electric resonance frequency $\omega_{e}$. Equally, the same geometry may be viewed as two close by electric short wire resonators (resonant linear electric current oscillating along the finite wire determined by the wire's self-inductance and the capacitance between it's ends) which couple such that the resonance splits into two modes which have either anti-parallel linear currents (magnetic resonant mode; finite magnetic moment, zero electric moment) or parallel currents (electric resonant mode; zero magnetic moment, finite electric moment) in the adjacent short wires. The parallel short wires can again be considered as LC circuit; the inductance composes of the straight wire self- and mutual inductance (and at upper THz frequencies, the contribution from the electron mass), the capacitance is given by the capacitance between the wires and that from end to end of each short wire. If this basic resonator element is arranged in a periodic array to form a metamaterial we have additional coupling between adjacent unit cells. Qualitatively, we can distinguish two limiting cases which are shown in Fig. 8 (left) and Fig. 8 (right). These designs have the advantage over SRR based metamaterials that magnetic response and a negative refractive index can be achieved for normal incidence to the layered structure. For SRR based designs propagation in the SRR plane is required. This becomes essential for high frequencies where layered structures are readily available to the experiment but true volume metamaterials are very complicated to achieve.

In the case of separate short wire pairs shown in Fig. 8 (left), the short wires couple in longitudinal direction capacitively. The response is magnetic resonant and electric resonant; the coupling capacitance can then be used to shift the electric resonance frequency relative to the magnetic resonance and make the negative responses overlap $^{29}$ to achieve a negative effective index. Metamaterials made of a single double-layer of short-wire pairs have been fabricated and demonstrated to have resonant magnetic response in the upper THz region. ${ }^{16}$ Although the reduction to a very simple design providing magnetic and electric resonant response simplifies fabrication these structures have the tendency to have huge losses, attributed in part to employing two resonances with the associated resonant losses. An alternative coupling of the short-wire pair in a periodic metamaterial is to introduce "thin" interconnects between the short wires of adjacent unit cells in longitudinal direction, hence 

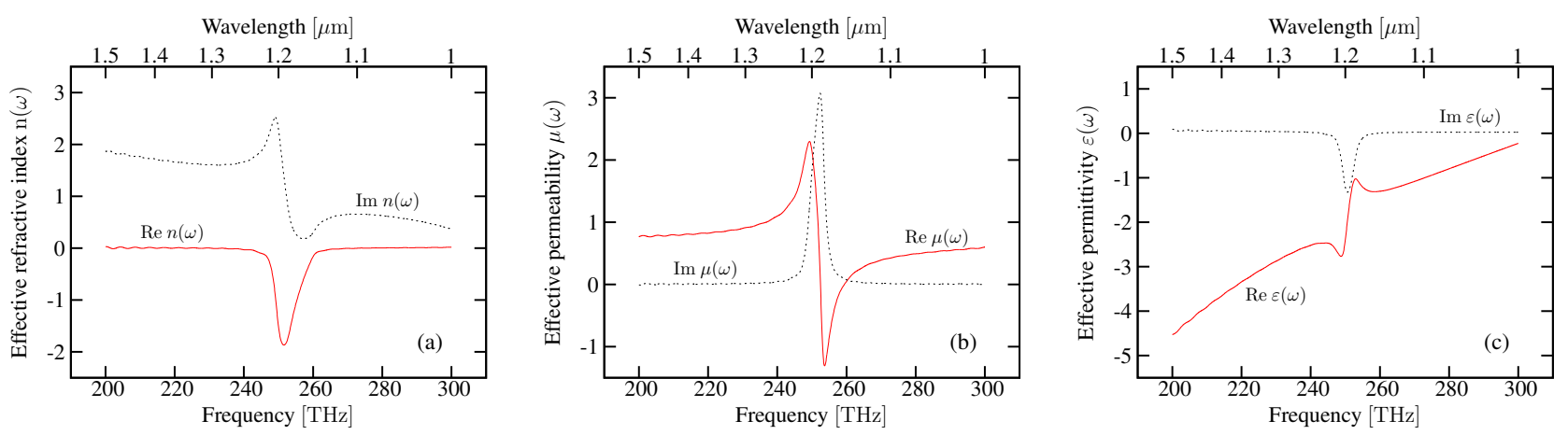

Figure 9. Retrieved real and imaginary part of the effective index of refraction (a), effective permeability (b), and effective permittivity (c) for a fishnet structure like shown in Fig. 8 (right). The parameters used are $a_{x}=a_{y}=600 \mathrm{~nm}$, $a_{z}=205 \mathrm{~nm}$ for the size of the unit cell; $w_{x}=270 \mathrm{~nm}, w_{y}=120 \mathrm{~nm}, t=60 \mathrm{~nm}, s=45 \mathrm{~nm}$, and a dielectric constant $\varepsilon_{\text {substrate }}=2$ for the dielectric layer. The metal is gold, describes by a Drude model dielectric function $\left(\omega_{p}=2175 \mathrm{THz}\right.$, $\left.\omega_{\tau}=6.5 \mathrm{THz}\right)$.

facilitating an inductive instead of an capacitive coupling. Conveniently, the width of the short wires in $\mathrm{H}$ direction can be extended across the unit cell to enhance the magnetic response (volume filling factor). The result of this operation is the "fishnet" structure ${ }^{31}$ shown in Fig. 8 (right). Here, the wide block $w_{x} \times a_{y}$ is what remains from the short-wire pair, the thin bridges in E-direction are the inductive interconnects. This structure exposes the magnetic resonant response of the short-wire pairs (here as anti-parallel currents in E direction in the wide blocks) but the (lowest) electric resonant is replaced by a electric plasmonic response due to the replacement of the longitudinal coupling capacitance by a coupling inductance. As a simple image, one may imaging this as a combination of short-wire pair (magnetic resonance) with continuous wires (providing the electric plasmonic response). The missing electric resonance tend to reduce the losses in this case.

In Fig. 9 we show the retrieved effective parameters for such a "fishnet" structure working in the near infrared frequency range. The resonant response in $\mu(\omega)$ reaching $\mu \approx-1$ near $255 \mathrm{THz}$ and the plasmonic negative permittivity are clearly visible. The anti-resonant spectral feature in $\varepsilon(\omega)$ around the magnetic resonance frequency $\omega_{m}$ and the accompanying negative imaginary part are periodicity artifacts ${ }^{24}$ caused by the relatively small wave length over unit cell size ratio of only $\approx 6$ in this case.

\section{CONCLUSION}

Using detailed numerical simulations we have systematically studied the scaling of the resonance frequency $\omega_{m}$ of single- and multi-gap SRRs in the THz region. It has been found that $\omega_{m}$ scales inversely proportional to the size $a$ of the unit cell of the SRR at microwave and lower THz frequencies. Above these frequencies, $\omega_{m}$ saturates. The saturation value depends on the geometry and the number of SRR gaps; for single-gap SRR around 100 $\mathrm{THz}$, for 4-gap SRR $500 \mathrm{THz}$ can be reached. The reason for the saturation is due to the increased kinetic inductance of the electrons (compared to the geometric inductance of the SRR ring). We have shown that losses scale linearly with the resonance frequency until the saturation of $\omega_{m}$. For higher frequencies losses saturate too and eventually decrease slightly as the resonance dies out. The non-resonant background loss increases in all regions with frequency. We found negative effective permeability up to $500 \mathrm{THz}$ in the 4 -gap SRR design. The saturation of the resonance magnetic frequency, as well as the losses, are responsible for the disappearance of the negative magnetic SRR response. We have shown that the SRR also provides a non-resonant diamagnetic response which differs below and above the magnetic resonance as either only the metal area of the SRR ring or the whole enclosed area of the SRR contribute. The diamagnetic response can explain the derivation of $\mu(\omega)$ from unity which is observed at low and high frequencies. We studied higher order excitations or plasmon modes which occur at $\mathrm{THz}$ frequencies. Consideration of the charge-density waves on the SRR ring is important to understand the behavior. Finally, we discuss two previously suggested alternative metamaterial designs and explain their relation in a unified picture. 


\section{ACKNOWLEDGMENTS}

Work at the Ames Laboratory was supported by the Department of Energy (Basic Energy Sciences) under Contract No. DE-AC02-07CH11358. This work was partially supported by the AFOSR under MURI grant (FA9550-06-1-0337), by DARPA (Contract No. MDA-972-01-2-0016), by Department of the Navy, Office of Naval Research (Award No. N00014-07-1-0359), EU FET projects Metamorphose and PHOREMOST, and by Greek Ministry of Education Pythagoras project.

\section{REFERENCES}

1. V. G. Veselago, Sov. Phys. Usp. 10, 509 (1968).

2. For recent review see C. M. Soukoulis, M. Kafesaki, and E. N. Economou, Advanced Materials 18, 1941 (2006); C. M. Soukoulis, Optics and Photonics News, June 2006, p. 16.

3. R. A. Shelby, D. R. Smith, S. C. Nemat-Nasser, and S. Schultz, Appl. Phys. Lett. 78, 489 (2001).

4. D. Smith, W. Padilla, D. Vier, S. Nemat-Nasser, and S. Schultz, Phys. Rev. Lett. 84, 4184 (2000).

5. J. B. Pendry, Phys. Rev. Lett. 85, 3966 (2000).

6. J. Pendry, A. Holden, W. Stewart, and I. Youngs, Phys. Rev. Lett. 76, 4773 (1996).

7. J. Pendry, A. Holden, D. Robbins, and W. Stewart, IEEE Trans. Microwave Theory Tech. 47, 2075 (1999).

8. K. Aydin, K. Guven, M. Kafesaki, L. Zhang, C. M. Soukoulis, and E. Ozbay, Opt. Lett. 29, 2623 (2004).

9. C. G. Parazzoli, R. B. Greegor, K. Li, B. E. C. Koltenbah, and M. Tanielian, Phys. Rev. Lett. 90, 107401 (2003).

10. R. A. Shelby, D. R. Smith, and S. Schultz, Science 292, 77 (2001).

11. C. Enkrich, M. Wegener, S. Linden, S. Burger, L. Zschiedrich, F. Schmidt, J. F. Zhou, T. Koschny, and C. M. Soukoulis, Phys. Rev. Lett. 95, 203901 (2005).

12. N. Katsarakis, G. Konstantinidis, A. Kostopoulos, R. Penciu, T. Gundogdu, M. Kafesaki, E. N. Economou, T. Koschny, and C. M. Soukoulis, Opt. Lett. 30, 1348 (2005).

13. S. Linden, C. Enkrich, M. Wegener, J. F. Zhou, T. Koschny, and C. M. Soukoulis, Science 306, 1351 (2004).

14. T. J. Yen, W. J. Padilla, N. Fang, D. C. Vier, D. R. Smith, J. B. Pendry, D. N. Basov, and X. Zhang, Science 303, 1494, (2004).

15. N. Katsarakis, T. Koschny, M. Kafesaki, E. N. Economou, and C. M. Soukoulis, Appl. Phys. Lett. 84, 2943 (2004).

16. G. Dolling, C. Enkrich, M. Wegener, C. M. Soukoulis, and S. Linden, Opt. Lett. 31, 1800 (2006).

17. G. Dolling, C. Enkrich, M. Wegener, C. M. Soukoulis, and S. Linden, Science 312, 892 (2006).

18. J. F. Zhou, T. Koschny, L. Zhang, G. Tuttle, and C. M. Soukoulis, Appl. Phys. Lett. 88, 221103 (2006).

19. J. F. Zhou, L. Zhang, G. Tuttle, T. Koschny, and C. M. Soukoulis, Phys. Rev. B 73, 041101 (2006).

20. G. Dolling, M. Wegener, C. M. Soukoulis, and S. Linden, Opt. Lett. 32, 53 (2007).

21. C. M. Soukoulis, S. Linden, and M. Wegener, Science 315, 47 (2007).

22. J. F. Zhou, T. Koschny, M. Kafesaki, E. N. Economou, J. B. Pendry, and C. M. Soukoulis, Phys. Rev. Lett. 95, $223902(2005)$.

23. M. W. Klein, C. Enkrich, and M. Wegener,C. M. Soukoulis, and S. Linden, Opt. Lett. 31, 1259 (2006).

24. T. Koschny, P. Markos, E. N. Economou, D. R. Smith, D. C. Vier, and C. M. Soukoulis, Phys. Rev. B 71, 245105 (2005).

25. D. R. Smith, S. Schultz, P. Markos, and C. M. Soukoulis, Phys. Rev. B 65, 195104 (2002).

26. T. Koschny, P. Markoš, E. N. Economou, D. R. Smith, D. C. Vier, and C. M. Soukoulis, Phys. Rev. B 71, 245105 (2005).

27. S. Tretyakov, Metamaterials 1, 40 (2007).

28. K. Aydin, I. Bulu, K. Guven, M. Kafesaki, C. M. Soukoulis, and E. Ozbay, New Journal of Physics 7, 168 (2005).

29. J. F. Zhou, E. N. Economou, T. Koschny, and C. M. Soukoulis, Opt. Lett. 31, 3620 (2006).

30. V. M. Shalaev, W. S. Cai, U. K. Chettiar, H. K. Yuan, A. K. Sarychev, V. P. Drachev, and A. V. Kildishev, Opt. Lett. 30, 3356 (2005).

31. S. Zhang, W. Fan, N. C. Panoiu, K. J. Malloy, R. M. Osgood, and S. R. J. Brueck, Phys. Rev. Lett. 95, 137404 (2005). 